Masuma G. Mammadova, Zarifa G. Jabrayilova

DOI: 10.25045/jpit.v08.i1.09

Institute of Information Technology of ANAS, Baku, Azerbaijan

depart15@iit.ab.az

\title{
DEVELOPMENT PROBLEMS AND PROSPECTS OF THE MEDICAL EXPERT SYSTEMS
}

The paper provides information on medical expert systems (ES), and deals with the transformation problems of the natural medical intelligence into the artificial medical intelligence. It describes the capabilities of modern ES and development prospects. This paper also analyzes the respective situation in Azerbaijan, and shows the necessity of development of knowledge engineering and training the knowledge engineers for the medical sector in order to create e-health in Azerbaijan. It proposes to strengthen the activity in the field of implementation of the developed ES.

Keywords: medical expert systems, knowledge extraction techniques, aspects of data acquisition, knowledge engineering.

\section{Introduction}

Since the development of information technology, healthcare is one of its widely applied areas. It is known that doctors' performance is based on the synthesis of knowledge and experience of the previous generation of specialists. The rapid increase in knowledge, the improvement of the diagnostic methods and the trends toward narrow specialization observed in the modern healthcare in recent decades, makes it difficult to make adequate decisions in terms of the abundance of information. Therefore, to ensure the adequacy of medical decisions made in various areas of medicine and to enhance their effectiveness, it is urgent to introduce modern mathematical methods and artificial intelligence technologies, as well as the innovative approaches. Using these tools, the introduction of developed intelligent ES in medicine ensures more effective results. The main advantage of these systems is that they include the professional knowledge and experience of specialists and experts, and assist and support physicians in making decisions about the diagnosis and treatment of certain diseases.

The globalized world is completely believed to be the century of information; since 2012,2.5 Exabyte ( 2.5 x1060 bytes) of information has been generated a day in the entire world, which means the beginning of the century of "scary big data" [1]. Taking into account that medicine is a big area, which produces large amounts of data, and the fact that, according to the latest research, $30 \%$ of whole data collected and stored in the world is health data [2].Moreover, by 2020, the medical data is forecasted to be 25000 petabytes [3], one can imagine how difficult it will be for doctors to make decisions in this information flood. Although information sources were previously few, now their number has extremely increased. Today, there are 100 thousand treatment methods in medicine, and treatment method is chosen on the basis of information received about the patient. Currently, the number of laboratory studies is increasing by geometric progression [5], whereas this figure in the past 0.5 million a year and increased by 2 times every 4-5 years [4].

One of the problems arisen by the abundance of information in medicine is due to medical mistakes. It is indicated in [5] that the number of deaths due to doctors' mistake varies between 100 thousand (for comparison, it is like an airplane crash a day) in the USA, where 15-18\% of GDP is allocated to healthcare. While in Germany this figure varies between30-60 thousand (in other words, imagine that a small town of Germany is sinking every year). Nevertheless, these mistakes are not intentional or because of the doctors' irresponsibility or non-professionalism. The main part of the doctors' mistakes is related to medicines, which are wrongly appointed. Boston clinic reports that, today, there are more than 10 diseases and disease syndrome in the world, and more than 4 thousand medicines. There is a correlation between 2 thousand of them, which limits the possibility of their combined use; furthermore, 300 different radiological procedures and 1100 laboratory analyses exist [7]. Therefore, the doctor "sinking" in this flow of information makes 
approximate decision about the patients choosing thousands of information, and the rest of the information are neglected by the doctor, because, of course, a human is not able to remember more than 7 indicators at the same time and put forward a judgment [8].

Of course, the impossibility of keeping in mind such a volume of information and the abundance of information in the process of diagnosis and treatment leads to the problems of humanity. Taking into account the decisions made under time pressure at decisive moment, and the fact that any mistake may cost a human life (the patient's treatment is considered here), the importance of ensuring physicians with modern tools, which supports decision-making, can be comprehended. Depending on the nature of the issues to be decided in the medical sphere, medical information-retrieval systems, forecasting, information and surveillance, management, diagnostic, monitoring ES and others have been developed, without of which it is impossible to imagine modern medical systems. Each of these systems has its own appointment, certain structure, organizational and action principles, theoretical, algorithmic and instrumental base. Today, we should focus on ES in the field of healthcare, which are aimed at the diagnostics and treatment, monitoring issues and provide their successful solution.

This paper focuses on the urgency of the establishment of ES, its state of the art, development trends, and the problems of knowledge engineering. It also analyzes the situation of establishing ES in Azerbaijan and puts forward proposals.

\section{Technology for establishing ES}

The term of "Expert System" was first coined by Feigenbaum E. in 1977. Its essence is: "Involving expert knowledge of the principles and tools in the field of Artificial intelligence (AI) into the solution of practical issues, which are difficult to be formalized" [9]. In other words, ES has the following characteristics, and therefore are subject to the resolution of issues that cannot be formalized:

- The issues that cannot be expressed in numerical form;

- input data and the knowledge about the subject area is ambiguous, imprecise and contradictory;

- the purpose of the issue can not be displayed with well-defined goal function;

- the problem does not have an exact and clear algorithmic solution.

Dendral - the first ES was developed at late 1960s at Stanford University by Feigenbaum E.[10]. This system defined the organic structure of molecules on the basis of the spectrographic data of its chemical connections. Referring to the heuristic knowledge of expert chemists, who created knowledge base of ES, it was possible to find the decision right just with a couple of efforts out of million cases. The principles and ideas that form the basis of Dendral system were so effective that, even today, the chemical and pharmaceutical laboratories around the world are using them. The ideas and principles of the system realization are the basic principles of the recognition of images, which is one of the main areas of AI.

Mycin ES was developed at Stanford University in mid 1970s, and decision-making problem was first solved on the basis of incomplete information [11]. This system uses the knowledge of medical experts for the diagnosis and treatment of meningitis and diseases of bacterial blood infections. The trials of the system, namely, the rules of the knowledge base were based on logical principles reflecting the specificity of the subject. The development method of Mycin ES is still being used as the basic principles of modern ES.

Successful results of Dendral and Mycin systems have stimulated the solution of difficultly formalized issues referred to expert knowledge with the use of all guidelines and tools in various spheres, consequently, the era of the development of ES aimed at addressing diagnosis, identification, management, forecasting, planning, monitoring, design and other issues has started. In general, the development criteria of ES in any field are defined by the followings:

1. Data and knowledge is valid and does not change over time; 
2. The space of possible decisions is finite and not so wide;

3. Formal judgments are used in the problem solution;

4. To resolve the issue in accordance with their knowledge and shaping method, which is described in this knowledge will tell you at least have to be an expert.

In most cases, the technology for ES establishment is called Knowledge Engineering. The experts, who set up this process, are called knowledge engineers, and they are closely interacting with one or few experts in specific subjects. A knowledge engineer acquires actual knowledge about the subject area and obtains the procedure, strategy and empirical rules needed to resolve the issue from the experts and establishes ES based on the gained knowledge (Figure 1) [12].

Requests, problems

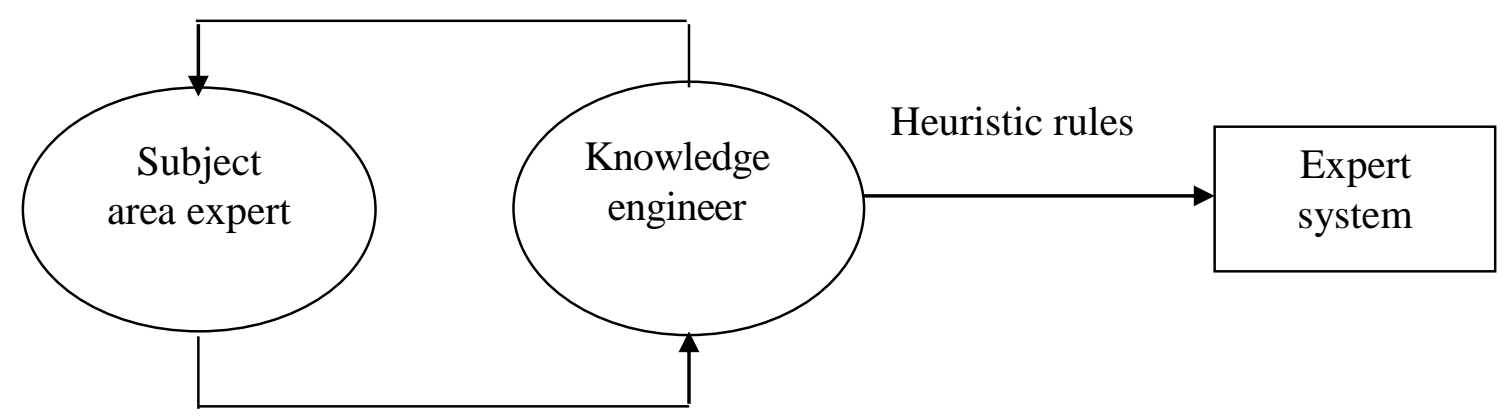

Solutions

Figure 1.Acquisition of knowledge from the experts for the ES establishment

\section{The issues of transforming natural health intelligence into artificial health intelligence}

\subsection{Methods of knowledge acquisition}

As mentioned, one of the main problems of ES establishment is to obtain knowledge. The knowledge acquisition -is the complete information as possible obtained by the knowledge engineer for decision-making in the subject area. Subject area -is an object studied for the automation and organization in of the management. The strategy of knowledge acquisition is described in Figure 2 [12]. This strategy uses automated and non-computer methods for knowledge acquisition $[13,14]$.

Automated methods include Internet search engines (Google, Yahoo, Yandex, Rambler), Data Mining, and OLAP systems that provide discovery and detection of the knowledge of practical significance out of original data important for decision-making in various spheres of human activity. The methods of knowledge acquisition without computer include communication methods and text-logical methods.

Communication methods refer to the methods of round table and brain attack. The round table discusses any problem with the participation of experts of the same rights. The participants first express their views, and then start the discussion. The participants prepare for the discussion in advance. Whereas, in brain attack, they do not prepare beforehand. The main goal of brain attack is the creation of ideas on the subject. After being generating a few ideas are analyzed, selected and promising ones are developed. 


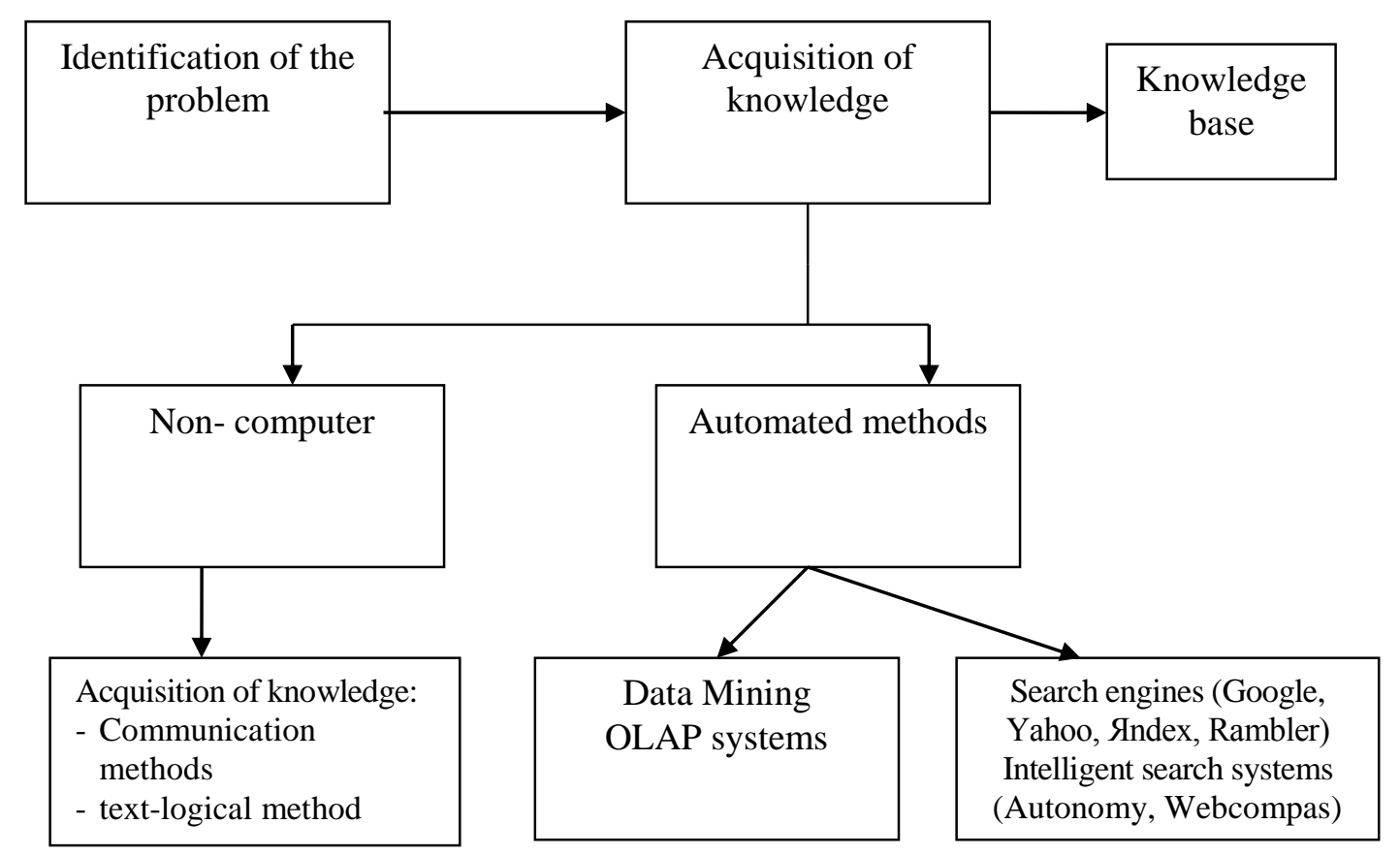

Figure 2. The strategy of knowledge acquisition

During the interview, which is one of the communication methods, the knowledge engineer prepares the questions in advance.

Unlike the questionnaire, during the interview, knowledge engineer may exclude or include some questions depending on the situation, and/or he/she can use different approaches to interest an expert and so on.

The dialogue does not include time limit and the questions are not prepared in advance.

The game of roles and the game of experts also refer to the communication methods. In the game of roles the roles are distributed across the participants (experts). Then, they are given a certain event (situation) and the decision-making process is being observed. In the game of experts a knowledge engineer watches the decision-making process in specific cases under the supervision of experts. Such games often use training simulators and computer systems.

Text-logical methods are associated with the analysis of regulatory and questionnaire materials related to the problem, orders, methodical manuals, instructions and other specific documents related to the subject. This method is based on the acquisition of the problem-related texts from the books, monographs, articles and other professional carriers of knowledge. The main highlights of understanding the text are: the formulation of the hypothesis about the entire text in advance (prior experience); identification of the substance of unclear concepts (particular terminology); defining a common hypothesis about the content of the text (of knowledge); specification of the content of terms (from complete to sections); establishing internal communication between the separate key words and fragments; adjustment of the common hypothesis (correction from sections to complete); adoption of the main hypothesis.

\subsection{Aspects of knowledge acquisition}

There are three aspects of the acquisition of knowledge, namely, psychological, linguistic and gnoseological aspects $[15,16]$.

The psychological aspect is the key aspect that ensures successful and effective interaction between a knowledge engineer and a source of knowledge - an expert. Less loss of information during the conversation, and high level communication between an analytics and expert depends on the psychological knowledge. The process of knowledge acquisition during the communicating 
can be described as the process of joint search for the truth. The communication model covers the participants, communication tools and communication object (knowledge). Depending on these components, three levels of psychological problem are: contact, procedures and cognitive.

In the contact level, efficiency of the communication between the experts and analyst depends on their gender, personal temperament, and the motivation of the communication participants. It is defined that better result is achieved if the experts and analysts are heterogeneous couple (male/female) and when the age relationship is as follows:

$5<($ EA - AA) $<20$, (where EA- expert's age, AA- analyst's age)

Linguistic aspect. The main problem in the field of linguistic aspect is related to the concepts: general code, concepts' structure, and user dictionaries. The general code is the intermediate communication language of between the cognitologyst and expert. This language incorporates the scientific and special concepts of professional literature. This eliminates the language barrier between the cognitologyst and expert. Subsequently, the general code turns into the structure of concepts or semantic network, which connects the concepts stored in the human memory. The user does not need to know the professional language in the subject area, so that the user dictionary is developed, which is also worked out on the general code.

Gnoseological aspect. This aspect combines the problem of acquisition of new scientific knowledge, so that the process of cognition is often observed with the creation of new concepts and theory. The experts form of several laws basing on the empirical experience accumulated in the development of a knowledge base. Gnoseological chain can be expressed in the following sequence:

"fact - generalized fact - empirical regularity - theoretical law".

Cognitologyst is interested in empirical knowledge of the experts, and they cannot be agreed upon. Knowledge is characterized by the following aspects:

- Systematicity (shows the location of new knowledge in multilevel structure);

- Knowledge objectivity (it is practically impossible to determine);

- Incomplete knowledge (impossibility of depicting the arbitrary subject field);

- Knowledge historicity (associated with 95\% growth or change in perceptions about the subject area over time).

At the initial stage, the cognitologyst uses decision structure and the various theories of experts to build a formal model of knowledge.

\subsection{Description models of knowledge}

Description model of knowledge is to present the knowledge obtained from the experts in the form of certain rules. Description models of knowledge re-classified in ES as follows [17-19]:

- Production model;

- Semantic network model;

- Frame model;

- Formal logic model;

- Relational model.

Production model is a rules-based model that describes the knowledge in the form of "If (condition), then (result)". "Condition" is such a sentence, on the base of which the search is enabled in the knowledge base, and the "result" is a situation obtained in the successful course of the search.

Semantic network is an oriented graphics, and its hills express the concepts, while the definitions express the relations between the concepts. The concept Any object denotes any concept, and the relation - the links between the objects. This model was proposed by American psychologist Kuillian.

Relations in the semantic network include:

"part-full" type relationship (class-group, element-set);

functional contact ( "occurs", "impact" and etc.); 
- $\quad$ Quantitative relationship (more, less, equal, etc.);

- $\quad$ Spatial relationship (far, near, under, above, inside etc.);

- $\quad$ Attribute relationship (has features, has essence etc.);

- $\quad$ Logical relationships (and, or, no);

- $\quad$ Linguistic relationships and etc.

Frame model was proposed by Marvin Minsky in 1970 for the perception of the visual (spatial) view of knowledge. Frame is a unit of knowledge description, and its details may vary according to the current situation. Frame is the minimum possible description of any event, situation, process or object. The traditional structure of frame is as follows:

(Frame name:

(1st slot name: 1 st slot essence)

(2nd slot name: 2nd slot essence)

(N-th slot name: $n$-th slot essence).

In the next step, each slot becomes the frame itself and derivates, so that this model is sometimes called tree model. For example, if we look at the room as a frame, its slots will be doors, windows, floor and ceiling. At the next stage, each slot can be viewed as the frame. In other words, if the frame is the door, its slots will be wood, iron, glass and etc.

Logical model of the description of knowledge based on the formal logic and predicate logic, and on the mechanism of logical conclusion of a human.

In the logical model of the descriptions of knowledge, the logical conclusion can be achieved based on 4 main these of the formal logic:

1. Modus Ponendo ponens:

If the implication $\mathrm{A} \rightarrow \mathrm{B}$ is true and $\mathrm{A}$ is true, then $\mathrm{B}$ is also true.

2. Modus Tollendo Tollens:

If the implication $\mathrm{A} \rightarrow \mathrm{B}$ is true, $\mathrm{B}$ is wrong, then $\mathrm{A}$ is also wrong.

3. Modus Ponendo Tollens:

If $\mathrm{A}$ is true and the conjunction $\mathrm{A} \wedge \mathrm{B}$ is wrong, then $\mathrm{B}$ is also wrong;

4. Modus Tollendo Ponens:

If $\mathrm{A}$ is wrong and the disjunction $\mathrm{A} \vee \mathrm{B}$ is true, the $\mathrm{B}$ is also true.

Relational model of the knowledge description is built according to features, performance, characteristics, in a word, criteria of the object.

\section{Modern medical ES and their application trends}

Today, ES is successfully implemented in medical field. There are thousands of ES in different fields of medicine in the world [20, 21]. However, the acquisition of these systems is almost impossible, as they are very expensive. Let's review brief information about widely used ES below:

> WebMD Symptom Checker system. The patients can get detailed information by uploading into the system the symptoms associated with the diseases related to allergies, arthritis, cancer, colds, flu, cough, depression, diabetes, eye diseases, heart disease, skin problems, sleep disorders. The system even allows the patients printing a report for submission to the doctors (offering advice, diagnosis, treatment) [22].

DXPlain is an example of intelligent clinical decision support system used to assist diagnostic process, and has a knowledge base that interconnects a list of diagnosis symptoms, laboratory data and procedures [23]. This is a software product of Massachusetts General Hospital.

INTERNIST is a consultant system for the diagnostics of internal diseases [21]. INTERNIST-1 is a modified version of the system and widely applied today [24]. 
CASNET is designed for glaucoma diagnosis and choice of treatment strategy. It is developed at Rutgers University in New Jersey, USA. It is based on the model of the semantic description of knowledge [21].

D EMYCIN - is used for diagnosis and treatment of infectious diseases of blood, and it is an improved version of Mycin [21].

$>$ Germwatcher ES is designed to detect, monitor and study the patients with infectious diseases in hospital;it was developed in 1993 at Washington University in St.Louis [25].

PEIRS interprets and explain the reports on the chemical pathologies [26].

$>\boldsymbol{H E L P}$ - complete hospital information system based on artificial intelligence technology, which supports not only standard functions of a hospital information system, but also decision support functions. AppHelp system offers automated judgments to take preventive measures during the acute pain in the abdominal cavity in the case of uncertainty. This system, developed for the diagnosis of acute pain in the abdominal cavity in 1972 at the University of Leeds (UK).

> PIP system - developed at the Technical University of Massachusetts is a software product designed on the basis of information collected and generated associated with the kidney defects at Tufts-New Medical Center in England [21].

In general, at present, medical ES and decision support systems based on the methods and principles of AI technology are used in various fields of clinical healthcare. The ES designed for clinical healthcare fields are applied in the following issues:

$>$ Providing alarms and reminders. The ES designed for this purpose provides the realtime monitoring of the patients through bed-mounted monitor, i.e., tracks the basic parameters of the patient's condition after the operation. ES installed in the monitors evaluates the changes in the condition of patients and also reminds the importance or the rules of taking medicines (for example, sending reminders via e-mail).

$>$ Assisting the process of diagnosis. Such ES assists and supports a doctor in complex situations to study the information about the patient and appoint diagnosis, who does not have enough experience.

$>$ Search for the appropriate conditions (precedents). This search can be conducted on the Internet or a local database. Such an intelligent system (agent) can choose appropriate into having the knowledge about the main characteristics of the patient's condition.

$>$ Therapy control and planning. This intelligent system is capable to control incompleteness, errors in the course of available treatment or the cases, when insufficiently taking into account the specific characteristics of the patient.

$>$ Recognition and interpretation of images. A large number of medical images are automatically interpreted: ranging from X-ray images to complex tomography images.

$>$ Monitoring clinical and pharmacological properties of drugs (toxicity). The system is aimed at detecting side effects and inconsistencies of the drugs. It models clinical symptoms and the doses of taken drugs. The system fulfills the monitoring of the treatment process to control the use of drugs.

\section{The development of medical ES in Azerbaijan}

A number of ES have been established in Azerbaijan in the field of medicine. Hence, in 1996, ES was established for the diagnosis of the acute surgical diseases of the members of abdominal cavity; ES was performed in clinical conditions and the development of knowledge base for the subject area [27]. The main objects and their characteristics of the subject field have been defined for the establishment of ES, diagnostic decision-making process has been analyzed, the main factors have been identified, the conceptual scheme of the subject field, and the structure of the database and knowledge base has been designed, the model for the solution of formal 
description of expert knowledge and diagnostic issues has been developed, ES's software has been developed and tested in real clinical hospital environment. The system is built with Lisp programming language.

Taking into account the mistakes made in the diagnosis of acute surgical diseases of the members of the abdominal cavity in the clinical medicine, ES with higher quality and faster diagnostic capabilities was created in 2000 [28]. In particular, the efforts were made to eliminate as much as possible the factors making impossible the clinical interpretation of health data and contributing to the wrong decisions, i.e. the lack health data bank about the patients.

In 2001, intelligent system executing primary diagnosis and hospitalization of the patients in multidisciplinary hospital was developed; and using artificial intelligence techniques the methodology of developing the intelligent system was proposed, which realizes primary diagnosis in the multidisciplinary medical centers and can allocate the patients within departments [29]. The software product of the system has been developed for the Emergency Department of Baku City Clinical Hospital No.1.

In 2003, the method for ES development were presented, which examine the functional status of the thyroid gland and fulfills its functional diagnostics [30].

In 2004, the information and diagnostic system was developed for breast tumors, and differential diagnosis system - for breast tumors [31]. In the course of development, the following issues have been solved: studying computer system for the diagnosis of tumors of the mammary glands and research activities; examining a list of factors based on the opinions of a group of doctors and choosing the most important factors; initial data collection, storage and processing; generating numerical matrix - a diagnostic card for each type of tumor by the age groups; developing an heuristic differential diagnosis algorithm according to the diagnostic card and on the information about the specific patient; the differential diagnosis problem statement as a matter of classification and its interpretation in terms of the theory of neural networks; development, training and evaluation of two-leaved neural networks; comparative analysis of the results of the doctor's diagnoses and neural network; developing specific computer programs and information diagnostic system for mammary gland tumors.

In 2005, the intelligent system developed for the selection of the surgical intervention in orthopedics was first used for the creation of artificial vision of the source of pathology [32]. The snapshot generated through photo-robot allows studying the characteristics of the pathological source, their recognition and classification, and the visualization of consequences and results of the surgical operations. The signs and their frequency priorities have been first defined for the recognition of the pathological source; and the structural scheme defining the situations in accordance with the signs and identifying the treatment sequence through the surgical intervention was created. According to this scheme, ES has been developed for the knowledge base and automated recognition of its situations, as well as for the visualization of treatment methods.

[33] has developed software complex aimed at resolving the problems arisen from the examination of the ambulatory patients in the field of ophthalmology. The development of the system has resolved the following issues:

- ambulatory original data has been collected for the diagnosis of eye diseases, and the database created based on it;

- knowledge base has been developed based on the differential indicators and a number of health data in ophthalmology;

- dictionary block (Azerbaijani, Russian, Latin and English) of ophthalmic terms has been built in the system;

- educational section has been created in the system to contribute the students and doctorsinterns, which increases the effectiveness of the system;

- public service sector has been created to educate and warn the population about the diseases. 
500 patients were received with eye diseases by the ambulatory department ofeye diseases No4 of Baku City Clinical Hospital No1 have been involved to the studies conducted for the implementation of this system.

[34] describes the software and structure of the virtual ophthalmologist ES in details.

[35] provides the establishment technology of ES for the diagnosis of neurological diseases, and the followings have been implemented in the course of the system development: neurological diseases have been investigated and the system of symptoms identified, including the methodology of knowledge description obtained for the diagnosis of neurological diseases, the structure of the knowledge base, the mechanism of logical conclusion, fuzzy mathematical model providing diagnostic strategy, proper ES architecture, operating principles and instrumental tools have been developed. ES for the diagnosis of neurological diseases offers the knowledge description in 6 stages based on ontology [36]. Within the framework of the study, fuzzy logic and expert knowledge have been used to systematize the types of epilepsy, and a diagnostic ES has been created [37]. The established system has been tested in clinical conditions and $83 \%$ accuracy has been performed.

[38] presents the operating principle of the system created for predicting 2 type diabetes in the people suffering from obesity. The system has been developed on the basis of statistical data processing methods and used dispersion analysis method for this purpose.

\section{Conclusion}

Today, the establishment of ES is one of the urgent issues. This urgency stems from the adoption of correct decisions for the improvement of services provided to patients, the appointment of diagnosis, and the selection of the treatment method. It is one of the fields of e-health formation. These systems are established by referencing to artificial intelligence methods and innovative approaches in the world of extremely growing information, and therefore, they require the adoption of appropriate technologies, and the training of the experts in the relevant field - knowledge engineers. This urges the training of specialized professionals in the integration of medical field and ICT once again and brings up benefiting from the experience of emerging countries in the field of e-medicine [39].

Citing the ES mentioned in this article, we can state that the Republic of Azerbaijan has successful achievements in the field of ES establishment, and intelligent ES developed for the diagnosis, monitoring and selection of treatment methods for various diseases has been successfully tested in different health centers. However, such science-based systems established with innovative technologies have been successfully tested, they are not exploited. Obviously, establishment of ES is often considered to be an experimental area, and it is related to constantly upgrading the systems to ensure the adequacy of the systems, inclusion of new knowledge, adding a new block, and this is regarded as a step forward perfecting the system. As a result, the creation of commercial-level ES can be achieved and the quality of medical services provided to the people can be improved with the mass use of the system. Failure of the established systems and enhancing the scale of the experiments to provide their realization prevents the contribution of these systems to the public.

\section{References}

1. Mammadova M.H., Jabrayilova Z.G. Opportunities and challenges of the use of big data in human resource management issues // Problems of Information Technologies, 2016, No1, pp.39-48.

2. Manchini M. Exploiting Big Data for improving healthcare servuces// Journal of e-Learning and Knowledge Society, 2014, vol.10, no.2, pp.23-33.

3. The growth of information volume - realities of the digital universe. www.tssonline.ru/articles2/fix-corp/rost-obema-informatsii-realii-tsifrovoy-vselennoy

4. Anisimov V.E. Basics of Medical Cybernetics: Textbook. Voronezh: VSU Publishing House, 1978, $240 \mathrm{p}$. 
5. Big Data in Human Resource Management - Developing Research Context. www.researchgate.net/publication/275520745

6. Znamenskaya T. Why IT in health care? // Open systems, No02, 2010. www.osp.ru/os/2010/02/13001446/

7. Davenport T., Glaser J., Just-in-time delivery comes to knowledge management, Harvard Business Review, 2002, vol.80, no.7, pp.107-111, doi: 10.1225/R0207H.

8. Miller G. A. The magical number seven, plus or minus two: Some limits on our capacity for processing information // Psychological Review, 1956, vol.63, pp.81-97.

9. Feigenbaum E.A. The Art of Artificial Intelligence: I. Themes and Case Studies of Knowledge Engineering / Proceedings of the International Joint Conference on Artificial Intelligence, 1977, pp.1014-1029. file:///C:/Users/HP/Downloads/rh996br0518.pdf

10. Buchanan B. G., Feigenbaum E.A. Dendral and Meta-Dendral: Their Applications Dimension // Artificial Intelligence, 1978, vol.11, no.5, pp.5-24.

file:///C:/Users/HP/Downloads/ym915sk9042.pdf

11. Shortliffe E.H., Buchanan B.G. A model of inexact reasoning in medicine // Mathematical Biosciences, 1975, vol.23, no.3-4, pp.351-379. doi:10.1016/0025-5564(75)90047-4

12. Gavrilova T.A., Khoroshevsky V.F. Knowledge Base of Intelligent Systems, St. Petersburg.: Peter, 2000, $384 \mathrm{p}$.

13. Knowledge. Types of knowledge. http://daxnow.narod.ru/index/0-19

14. Knowledge engineering technology. www.iskhacov.narod.ru/materials/enginer.pdf

15. Theoretical aspects of knowledge extraction. http://lib.alnam.ru/book_bki.php?id=24

16. Strategy of acquiring knowledge. http://itteach.ru/predstavlenie-znaniy/strategii-polucheniyaznaniy

17. Davis R., Shrobe H., Szolovits P. What is a Knowledge Representation? // AI Magazine, 1993, vol.14, no.1, pp.17-33. http://groups.csail.mit.edu/medg/ftp/psz/k-rep.html

18. Introduction to Knowledge Modeling. http://www.makhfi.com/KCM_intro.htm

19. http://edu.dvgups.ru/METDOC/EKMEN/MEN/SIST_UPR/METOD/OSN_UPR/Klykov_25.htm

20. Karimov S. Rahimov N. Expert System. Baku, Çaşığlu, 2004, 173 p.

21. Randolph A. Miller, Harry E. Pople, Jr., and Jack D. Myers. INTERNIST-1-An Experimental Computer-Based Diagnostic Consultant for General Internal Medicine // New England Journal of Medicine. 1982, vol.307, pp.468-476.

http://people.dbmi.columbia.edu/ ehs7001/Clancey-Shortliffe-1984/Ch8.pdf

22. WebMD. http://symptoms.webmd.com/symptomchecker

23. The features of DXplain. http://dxplain.org/dxp2/dxp.sdemo.asp?login

24. Jadhav V.S., Sattikar A. A. REVIEW of Application of Expert Systems in the Medicine, pp.122-124. http://nci2tm.sinhgad.edu/NCIT2M2014_P/data/NCI2TM_31.pdf

25. The GermWatcher. www.openclinical.org/aisp_germwatcher.html.

26. PEIRS: a pathologist-maintained expert system for the interpretation of chemical pathology reports.www.ncbi.nlm.nih.gov/pubmed/8316495

27. Rahimova N.A. Expert system for the diagnosis of surgical diseases of the abdominal cavity, thesis. summary. Baku, 1996, p. 22.

28. Geybullayev A.A., Rahimova N.A., Habibullayev C.B. The development and the use of diagnostic expert systems for the acute surgical diseases of the abdominal cavity members. Baku, Elm, 2000, 173 p.

29. Abdullayeva G.G., Heydarov N.G., Valiyev E.S. and et.al. The intelligent system of primary sorting of patients in a multidisciplinary hospital // Proceedings of ANAS, Series of PhysicalTechnical and Mathematical Sciences, 2001, No2, pp.103-105.

30. Abdullayeva G.G., Mammadova M.V. Expert system of recognition of functional state of the thyroid gland in the cases of difficult diagnosis // Proceedings of ANAS, Series of PhysicalTechnical and Mathematical Sciences, 2003, No 2-3, pp.126-129. 
31. Abdullayeva G.A. The development of information-diagnostic system for breast tumors: thesis. summary. Baku, 2004, 20 p.

32. Hajiyev Z.A. Intelligent system for the choice of surgical intervention in Orthopedics. thesis. summary. Baku, 2005, 20 p.

33. Shukurlu S.F. expert system for the primary diagnosis of ambulatory patients in ophthalmology: thesis. summary. Baku, 2005, 20 p.

34. Musayev P.I., Shukurlu S.F. "Virtual ophthalmologist" expert system software and structure // Scientific and Practical Journal of Ophthalmology, 2009, No1, pp.24-29.

35. Amooji A.Sh. The establishment of expert systems for the diagnosis of neurological diseases: Expert System: thesis. summary.Baku, 2016, 18 p.

36. Aliguliyev R., Amooji A. Knowledge description models in the expert system for the diagnosis of neurological diseases / Proceedings of the First Republican scientific-practical conference on the "Multidisciplinary problems on e-health", Baku, May 25, 2015, pp.171-174.

37. Mammadova M., Amooji A. Expert system for the diagnosis of epilepsy // Proceedings of the First Republican scientific-practical conference on the "Multidisciplinary problems on e-health", Baku, May 25, 2015, pp.211-214.

38. Kurbanova N., Mustafayev A. Screening system for the prediction of $2^{\text {nd }}$ type diabetes with obesity / Proceedings of the First Republican scientific-practical conference on the "Multidisciplinary problems on e-health", Baku, May 25, 2015, pp.201-203.

39. Jabrayilova Z.G. Forming the human resources in E-medicine: international experience, solutions and perspectives // Problems of Information Society, 2016, No2, pp.61-73. 